\title{
Criminologie
}

\section{Où en est la situation de la violence envers les femmes dans le milieu familial, dix ans après les colloques sur la violence?}

\section{Ginette Larouche et Louise Gagné}

Volume 23, numéro 2, 1990

Après le crime : survivre

URI : https://id.erudit.org/iderudit/017293ar

DOI : https://doi.org/10.7202/017293ar

Aller au sommaire du numéro

Éditeur(s)

Les Presses de l'Université de Montréal

ISSN

0316-0041 (imprimé)

1492-1367 (numérique)

Découvrir la revue

Citer cet article

Larouche, G. \& Gagné, L. (1990). Où en est la situation de la violence envers les femmes dans le milieu familial, dix ans après les colloques sur la violence?

Criminologie, 23(2), 23-45. https://doi.org/10.7202/017293ar
Résumé de l'article

The article presents the situation regarding violence to women for the region of Montreal. The nature and extent of the problem is examined and the risk factors are dealt with from a multidimensional point of view. The community and para-public services associated with this problem are described briefly and an account is given of the impact of past and present action. Some future prospects are also introduced. 


\section{OÙ EN EST LA SITUATION DE LA VIOLENCE ENVERS LES FEMMES DANS LE MILIEU FAMILIAL, DIX ANS APRÈS LES COLLOQUES SUR LA VIOLENCE? \\ Ginette Larouche* \\ Louise Gagné *i*}

The article presents the situation regarding violence to women for the region of Montreal. The nature and extent of the problem is examined and the risk factors are dealt with from a multidimensional point of view. The community and para-public services associated with this problem are described briefly and an account is given of the impact of past and present action. Some future prospects are also introduced.

\section{INTRODUCTION}

En 1990, la violence envers les femmes demeure d'actualité. Les journaux font régulièrement état de drames familiaux, témoignant ainsi des diverses violences dans le milieu familial.

Cet article propose de faire un portrait de l'état de situation de la violence conjugale à Montréal et d'identifier la distribution régionale des services existants pour répondre à ce phénomène. Un bilan présentera l'impact des actions passées et présentes.

En plus d'aborder la violence conjugale, une réflexion sera amorcée afin d'identifier d'autres formes de violence faite aux femmes dans le milieu familial. Sans développer exhaustivement l'aspect de la violence à l'égard des aînés et des enfants, des parallèles seront établis. Cette démarche permet de faire émerger les diverses victimisations dans le milieu familial et d'aborder les facteurs de vulnérabilité de façon multidimensionnelle.

Dans un premier temps, la nature du problème sera brièvement décrite. Suivront des données statistiques sur l'ampleur de la violence conjugale à Montréal. Par la suite, certains facteurs de risques associés à un comportement d'agresseur et certains indices de tolérance à la violence chez les victimes seront identifiés. Cette réflexion se fera à partir de différentes catêgories de victimisation. Finalement, les services en violence conjugale disponibles dans

* M.Sc. en service social

** Conseil de la santé et des services sociaux de la région du Montréal métropolitain, 3725, rue St-Denis, Montréal, Québec, H2X 3L9. 
la région de Montréal seront nommés afin d'établir l'êvolution sur ce phénomène depuis les dix dernières années.

\section{NATURE DU PROBLÈME}

Depuis plusieurs années, la violence conjugale n'est plus considérée comme un phénomène individuel mais comme un problème d'ordre social '. Maintenant, il est bien connu que cette réalité touche toutes les classes sociales, toutes les nationalités, elle se rencontre en milieu urbain comme en milieu rural et affecte tous les groupes d'âge ${ }^{2}$. Les recherches ont démontré qu'on ne peut établir un portrait de la femme battue et de l'homme violent, puisqu'il n'y a pas de facteurs individuels qui permettent de déterminer un profil démographique ou personnel de l'agresseur ${ }^{3}$; il en est de même pour les femmes violentées ${ }^{4}$.

Les femmes sont sans contredit les victimes de la violence conjugale ${ }^{s}$. Cette violence se manifeste de différentes façons: agressions psychologiques, physiques, sexuelles et homicide ${ }^{6}$. Elle crée des blessures importantes aux plans physique, émotif et mental ${ }^{7}$. Sur le plan physique, les blessures nécessitent des soins médicaux dans près d'un tiers des situations'. De plus, 18 à $21 \%$ des femmes admises aux urgences chirurgicales dans les hôpitaux sont des femmes battues". D'autre part, les séquelles psychologiques, même si elles ne sont pas visibles, s'avèrent graves: anxiété, dépression, mésestime de soi ${ }^{10}$, syndrome de la femme battue. On mentionne que $65 \%$ des femmes violentées font appel à la psychiatrie" . Notons que dans $41 \%$ des situations, l'escalade de violence se termine par un meurtre'?

La violence conjugale n'est pas un nouveau phénomène. Elle se vit depuis des siècles. Elle n'apparaît pas uniquement parce que les rapports conjugaux se négocient différemment, bien que selon Bourgon les femmes revendiquent plus sans que les hommes aient modifié leur façon brutale de réagir ${ }^{13}$.

Les courants d'analyse de la violence conjugale convergent de plus en plus vers la notion de pouvoir. L'acte de violence est alors vu comme un geste de domination de la part de l'agresseur ${ }^{14}$, une façon d'établir son autorité et de régler les conflits sans avoir à négocier. C'est pourquoi l'on retrouve dans les programmes d'aide aux hommes violents une démarche de responsabilisation pour l'agresseur ${ }^{15}$. Également, il n'est pas étonnant de constater dans les recherches l'importance pour les intervenants d'avoir une vision non traditionnelle de la vie du couple pour être en mesure de dépister et d'intervenir en violence conjugale ${ }^{16}$.

Ce nouvel éclairage sur la nature du problème émerge des 15 dernières années alors que la société tentait de taire le problème en le réduisant à une 
notion de pathologie, à un silence en prétextant l'aspect «privé» de cette problématique. L'on confondait alors les séquelles de la violence aux causes de cette dernière ${ }^{17}$. Malheureusement, ces mythes demeurent encore dans la population et chez certains professionnels malgré les efforts de sensibilisation. Encore aujourd'hui, les femmes violentées ont honte de dénoncer leur condition de vie et il est essentiel de la dénoncer. Pour ce faire, les intervenants (médecins, infirmières, personnel psychosocial, policiers, etc.) doivent être formés pour dépister les cas de violence conjugale et intervenir adéquatement.

D'autre part, rappelons que la violence conjugale se vit également chez les personnes âgées. Soulignons que la violence envers les aînés a lieu généralement dans le milieu familial ${ }^{18}$ et s'exerce particulièrement à l'égard des femmes ${ }^{14}$. L'étude de Grandmaison, faite auprès d'une clientèle âgée des services sociaux du Montréal métropolitain, démontre que $22,8 \%$ des violences faites aux aînées dans le milieu familial est produite par le conjoint ${ }^{20}$. Les femmes âgées, en plus de vivre la honte, craignent d'être abandonnées ou placées si elles dévoilent leur victimisation.

En plus de connaître la violence conjugale, les femmes âgées vivent également l'agression de leurs enfants. Les fils ou les beaux-fils viennent alors au premier rang des agresseurs dans le milieu familial ${ }^{21}$. Elles connaissent des abus physiques, de la violence psychologique, de l'exploitation financière ou encore elles subissent de la négligence et ne reçoivent pas les soins qu'elles requièrent ${ }^{22}$. La quantification de ces violences n'est pas encore possible. Certains auteurs avancent que le taux de signalement des abus physiques serait de $4 \%$ mais qu'un seul cas sur six serait signalé ${ }^{23}$.

À partir des statistiques, il semble que les femmes demeurent les principales victimes des sévices physiques commis envers un adulte dans le milieu familial. Afin de compléter le portrait de la violence familiale, il faut ajouter les abus commis à l'égard des enfants. Ces données demeurent plus accessibles depuis l'existence de la Loi de la protection de la jeunesse au Québec.

La violence envers les enfants au Québec recouvre l'abus physique, sexuel et certains auteurs y incluent les enfants négligés. Pour l'année 1988-89, à Montréal, le taux de mauvais traitements dévoilés à la Protection de la jeunesse et retenus était de 17,5 par 1000 enfants et sur les 4031 enfants suivis dans les trois Centres de services sociaux du Québec, on compte 75,2\% de cas de négligence, $13,3 \%$ de situations d'abus physique et $8,5 \%$ de cas d'abus sexuels ${ }^{24}$. Les séquelles des violences chez les enfants sont évidemment nombreuses sur les plans émotif, physique et comportemental. Parmi les difficultés émotives les plus fréquemment mentionnées, on retrouve la faible estime de soi, l'autodépréciation ${ }^{25}$, l'anxiété excessive ${ }^{26}$, de la méfiance envers l'environnement ${ }^{27}$ et des troubles du sommeil ${ }^{28}$. Du côté des problèmes de 
comportement, les retards d'apprentissage ${ }^{24}$ se manifestent, des phobies scoJaires se présentent et les enfants ont également des comportements agressifs, principalement chez les garçons ${ }^{30}$.

La violence familiale, ou violence faite aux femmes et aux enfants, cause des difficultés importantes aux victimes. Le fait que cette violence ne soit pas toujours dépistée et traitée de façon spécifique pose donc des inquiétudes sur le plan social.

En effet, le dépistage et l'intervention en violence nécessitent des habiletés particulières puisque les victimes ont tendance à cacher leur réalité par honte ou par crainte de conséquences d'une dénonciation et qu'elles revendiquent encore peu des services d'aide.

\section{DIMENSION DU PROBLÈME}

Pour revenir à la violence conjugale, les données statistiques sur le phénomène demeurent encore pratiquement inexistantes. Elles sont morcelées et souvent fragmentaires puisque, de façon générale, il n'y a pas de données recueillies dans les organismes socio-sanitaires. Comme il se fait peu de dépistage auprès de cette clientèle, l'ampleur réelle reste sous-évaluée, bien que les chercheurs et intervenants s'entendent pour dire que la violence conjugale touche un grand nombre de femmes, qu'elle est un problème grave et répandu. Voyons quelques chiffres indicateurs.

Les experts consultés lors de la commission Rochon mentionnaient qu'une femme sur sept était violentée au Québec ${ }^{31}$. On rapporte également 300000 femmes ayant subi de la violence sévère au Québec en $1985^{32}$. Une étude canadienne soulève un taux de violence masculine dans un contexte conjugal de $13 \%$ au Québec ${ }^{33}$. Cette violence est produite par des hommes de 19 à 25 ans, ce qui est inquiétant pour la génération actuelle.

Malgré l'absence de statistiques à Montréal, si l'on croise les informations, on peut minimalement constater que, pour l'année 1988, il y a eu 3987 infractions relatives à la violence conjugale, une augmentation de $10,47 \%$ par rapport à l'année précédente ${ }^{34}$. De son côté, la cour municipale a reçu pour trois mois (juillet à octobre 1989) 135 dossiers de violence conjugale dont uniquement 10 ont eu un jugement de culpabilité ${ }^{35}$.

D'autre part, l'étude de Bennet ${ }^{36}$ mentionne qu'il y a eu en 1988 à la police de la C.U.M. 4557 appels de violence conjugale dont 1542 dossiers mentionnaient des voies de fait et 143 entraient dans la catégorie des agressions armées. Quatre postes de police sur six ont fait plus de 230 interventions policières et, toujours en 1988 , pour les mois de janvier à octobre, la police de 
la C.U.M. a porté 2494 accusations. Ce qui voudrait dire que $45 \%$ des appels ne se rendent pas à l'étape des accusations. Pour l'année 1989, les statistiques de la S.P.C.U.M. ${ }^{37}$ mentionnent qu'il y a eu 3481 infractions dont 2320 accusations, soit 174 accusations de moins que l'année précédente pour la même période. À première vue, la politique judiciaire ne semble pas avoir un impact réel sur les accusations. Le centre d'aide aux victimes d'actes criminels mentionne que sur 76 appels, 15 femmes violentées ont communiqué avec leurs services sur une période de sept mois (mars à septembre) en $1989^{38}$. Finalement, mentionnons qu'en $1983^{39} 53 \%$ des accusés se tiraient indemnes de leur accusation à la cour. La sentence moyenne d'un homme qui tue sa femme est de trois ans ${ }^{40}$. Il n'y a pas de statistiques permettant d'évaluer si la situation judiciaire a changé mais le secteur policier demeure une ressource importante pour les victimes.

Du côté des maisons d'hébergement, les statistiques sont partielles compte tenu des données non disponibles des maisons ne faisant pas partie du regroupement. Pour l'année 1988-89, les données du regroupement permettent d'avoir l'information pour la région métropolitaine". Ainsi, 794 familles ont été accueillies à Montréal pour cette année pour une moyenne de 99 familles par maison, soit deux femmes et quatre enfants de moins que l'année précédente. Mais les séjours sont plus longs. En effet, la moyenne est de 17 jours comparativement à 16 pour l'année 1987-88. En plus des hébergements et des services offerts lors de cette période, les maisons font en moyenne 54 consultations par le biais d'entrevues. Le taux d'immigrantes est de $13 \%$ et celles-ci sont majoritairement hébergées à Montréal. Finalement, 770 enfants majoritairement en bas âge ont été têmoins et/ou victimes de violence dans ces familles.

Du côté de S.O.S. Violence conjugale ${ }^{42}$, du 11 janvier 1987 au 26 mars 1989 , il y a eu 10586 appels téléphoniques. Plus de $50 \%$ de ces appels provenaient de la région de Montréal. La demande était pour de l'hébergement dans 2756 situations et pour de l'écoute et information dans 7830 des cas.

De façon complémentaire, on retrouve au C.S.S.M.M., 1283 dossiers d'enfants battus qui ont fait l'objet d'une prise en charge ${ }^{43}$, ce qui signifiait statistiquement 603 femmes violentées. Finalement, il est connu que le taux de violence prémaritale est de $20 \%$ et que $44,7 \%$ de ces relations se resserrent en un lien conjugal. Du côté des agresseurs, à titre indicatif, Option a reçu, pour l'année 1988, 490 hommes violents et S.A.C. a desservi 248 hommes agresseurs. 


\section{FACTEURS DE RISQUE}

En violence conjugale, on ne peut malheureusement pas identifier des facteurs de risque puisque les recherches ont démontré qu'aucune pathologie n'est précurseur d'un comportement de violence. Seulement $10 \%$ des agresseurs auraient un problème de santé mentale qui génère des comportements violents ${ }^{45}$.

La violence conjugale est le produit d'une société où le pouvoir conjugal était donné aux hommes (Code civil [articles 175-176]). Ce n'est que dans les années 80 que la loi sera modifiée avec la refonte du Code civil. L'emploi de la violence en soi est un moyen pour dominer un individu et établir ses propres règles, c'est un rapport de domination.

La question de risque doit donc être abordée de façon multidimensionnelle. Soulevons trois facteurs qui contribuent à la violence conjugale: les incitateurs envers les femmes, les «renforçateurs» de violence conjugale et ceux qui augmentent la tolérance des victimes à la violence. La première catégorie réfère à ce qui crée une relation de pouvoir entre les hommes et les femmes: l'enseignement des stéréotypes traditionnels aux enfants dans les écoles, dans la famille et dans les médias, le renforcement des rôles sexistes, la cellule familiale traditionnelle, la structure économique qui ne reconnaît pas la valeur de production des femmes ou qui prône la double tâche pour celles-ci; la pornographie, la violence à la télé, tout ce qui incite à user de violence envers les femmes. L'assemblée des Évêques du Québec (1989) ${ }^{\text {th }}$ dénonce un grand nombre de ces incitateurs à la violence. Ceux-ci favorisent ce que Fattah ${ }^{47}$ appelle la victimisation légitime des femmes dans notre société. C'est ce qui est à la base de la violence conjugale.

Le deuxième facteur renvoie aux renforçateurs de violence, l'homme abusif s'en sert pour justifier, minimiser et se déresponsabiliser de la violence qu'il produit. Dans ces renforçateurs, I'homme violent puise les appuis sociaux nécessaires au maintien de ses comportements de violence. Lc tablcau 1 résume ces facteurs.

Les données de ce tableau démontrent bien comment la société est encore tolérante à la violence conjugale et en assume encore les coûts (financiers, juridiques et sociaux). On cherche encore à justifier la violence, à l'analyser comme un problème intrapsychique alors que les recherches démontrent que tout homme peut s'autoriser à violenter sa conjointe s'il se trouve une justification.

Finalement l'indicateur de tolérance à la violence (tableau 2) renvoie aux victimes, à leurs zones de dépendance qui les rendent plus vulnérables dans 


\begin{tabular}{|c|c|c|}
\hline $\begin{array}{l}\text { L'ALLÉGEANCE AUX STÉRÉOTYPES } \\
\text { MASCULINS }\end{array}$ & $\begin{array}{l}\text { L'INTÉGRATION DE LA NOTION DU } \\
\text { POUVOIR }\end{array}$ & $\begin{array}{c}\text { DISCOURS ET MYTHES QUI RENFORCENT } \\
\text { LES ACTES VIOLENTS EN } \\
\text { DÉRESPONSABILISANT L'AGRESSEUR }\end{array}$ \\
\hline $\begin{array}{l}\text { - La virilité passe par des gestes agressifs et } \\
\text { violents } \\
\text { - L'homme perçoit sa partenaire comme un être } \\
\text { inférieur }^{49} \\
\text { - Intégration des normes masculines qui prônent } \\
\text { la violences } \\
\text { - La femme est vue comme un être qui doit } \\
\text { combler tous ses besoins affectifs } \\
\text { - La punition physique est un moyen légitime } \\
\text { pour contrôler sa partenaire } \\
\text { - L'agression sert à prouver sa domination et à } \\
\text { établir son pouvoir }\end{array}$ & $\begin{array}{l}\text { - Ex.: enfant témoin de violence }{ }^{\varsigma} \\
\text { - Ex.: enfant battu } \\
\text { - Emploi de la violence dans les relations } \\
\text { prémaritales }{ }^{\$ \dagger} \\
\text { - Bagarres avec les pairs durant l'enfance et/ou } \\
\text { l'adolescence } \\
\text { - Adolescents qui agressent leurs parents pour } \\
\text { avoir le contrôle } \\
\text { - La violence est perçue comme un moyen } \\
\text { acceptable de solutionner les conflits } s^{\varsigma 4}\end{array}$ & $\begin{array}{l}\text { - L'alcoolisme cause la violence } \\
\text { - L'homme violent est dépressif } \\
\text { - L'homme violent est stressé } \\
\text { - L'agresseur a un passé d'enfant battu } \\
\text { - L'agresseur a une pathologie ou un trouble de } \\
\text { la personnalité } \\
\text { - L'agresseur est jaloux de façon pathologique } \\
\text { - La violence des hommes est innée et a une } \\
\text { cause biologique (testostérone) } \\
\text { - Le stress et la fatigue sont des causes de } \\
\text { violence }\end{array}$ \\
\hline $\begin{array}{l}\text { Les stéréotypes traditionnels entretiennent les } \\
\text { comportements violents et généralement les } \\
\text { agresseurs y adhèrent pour justifier leur pouvoir } \\
\text { et leur droit de recourir à la violence. }\end{array}$ & $\begin{array}{l}\text { Les apprentissages qui permettent d'intégrer la } \\
\text { rentabilité de la violence pour solutionner des } \\
\text { conflits sans avoir à négocier. }\end{array}$ & $\begin{array}{l}\text { Rationnels qui servent à minimiser l'acte de } \\
\text { violence. Un homme pourrait présenter plusieurs } \\
\text { de ces problèmes sans être pour autant un homme } \\
\text { violent. }\end{array}$ \\
\hline
\end{tabular}


Facteurs qui renforcent la tolérance de la victime

\begin{tabular}{|c|c|c|}
\hline $\begin{array}{l}\text { LE STÉRÉOTYPE FÉMININ OU } \\
\text { L'INCAPACITÉ APPRISE }\end{array}$ & $\begin{array}{c}\text { FACTEURS PERSONNELS QUI } \\
\text { AUGMENTENT LA TOLÉRANCE } \grave{A} \text { LA } \\
\text { VIOLENCE }\end{array}$ & $\begin{array}{c}\text { DISCOURS ET MYTHES QUI } \\
\text { ENTRETIENNENT LA POSITION DE } \\
\text { VICTIME }\end{array}$ \\
\hline $\begin{array}{l}\text { - L'oubli de soi } i^{60} \\
\text { - La responsabilité de la nourriture affective dans } \\
\text { la cellule familiale } \\
\text { - La douceur et la passivitéét } \\
\text { - La censure de la colère }{ }^{63} \\
\text { - La faible estime de soi }{ }^{64} \\
\text { - L'apprentissage de la non-affirmation } \\
\text { - La croyance qu'une femme ne peut survivre } \\
\text { sans la présence d'un homme dans sa vie }\end{array}$ & $\begin{array}{l}\text { - Le passé d'enfant baltu ou témoin de violence } \\
\text { conjugale dans sa famille. la fillette devient } \\
\text { alors passive } \\
\text { - L'intégration du stéréotype féminin augmente } \\
\text { la tolérance à la violence. Elle a donc appris } \\
\text { à se sentir incapable } \\
\text { - La faible estime de soi. le manque de confiance } \\
\text { en soi, augmentent la tolérance à la violence } \\
\text { - La dépendance émotive } \\
\text { - La dépendance économique } \\
\text { - L'isolement social } \\
\text { - La dépendance psychologique (faible estime } \\
\text { de soi). L'importance de se réaliser à l'intérieur } \\
\text { d'une union } \\
\text { - La responsabilité de la famille } \\
\text { - L'abandon des décisions à son conjoint } \\
\text { - L Laugmentation des zones de dépendance par } \\
\text { l'intégration des stéréotypes féminins }\end{array}$ & $\begin{array}{l}\text { - La femme battue est masochiste, elle a un } \\
\text { comportement pathologique } \\
\text { - Elle ne veut pas s'en sortir puisqu'elle retourne } \\
\text { avec l'agresseur } \\
\text { - Elle contribue à la violence: elle la provoque } \\
\text { - Elle l'encourage en ne réagissant pas } \\
\text { - Dans un couple, il n'y a pas qu'un responsable } \\
\text { - L'homme est le maitre chez lui }\end{array}$ \\
\hline
\end{tabular}

Aucune caractéristique individuelie ne peut justifier la responsabilité de la femme battue dans son vécu de violence. L'intégration du stéréotype féminin de même qu'un vécu de violence dans l'enfance peuvent contribuer à augmenter le seuil de tolérance de la femme maltraitée. De surcrôt, les pressions sociales culpabilisent les femmes victimes. 
un contexte de violence et qui augmentent leur tolérance (colonne 2 du tableau 2) dans la relation conjugale. Notons ici que les femmes immigrantes connaissent une dépendance supplémentaire à leur communauté et que les femmes handicapées ont une contrainte de plus (pas de statistiques disponibles pour ces clientèles).

En fonction des risques de violence, il faut mentionner les besoins primaires des victimes d'être protégées (physiquement) de l'agresseur, leur besoin de recevoir de l'assistance financière, médicale, sociale et juridique. Ces besoins des femmes battues ne sont pas linéaires et successifs, ils se présentent souvent de façon complémentaire lors des situations de crise.

Actuellement, ces besoins ne peuvent être comblés que partiellement puisque chaque ressource existante a ses limites. De ce fait, aucune ressource ne peut répondre seule à l'ensemble des besoins exprimés par les femmes violentées. Les services qui s'adressent à ces femmes doivent viser à réduire les zones de dépendance à l'agresseur, à restaurer l'estime de soi et à augmenter les habiletés affirmatives. Il apparaît nécessaire que les organismes qui interviennent en «violence familiale» puissent offrir un service complet et adapté à la problématique, puisqu'on sait que seulement $10 \%$ des femmes violentées s'adressent à une ressource d'hébergement ${ }^{7 n}$.

Du côté de l'agresseur, celui-ci a besoin d'être responsabilisé de son geste (juridiquement), d'être dénoncé face à son abus de pouvoir (socialement) et d'être contraint à modifier ses comportements (aide psychosociale), d'être encadré (prévenir le suicide) pour modifier sa position d'agresseur. L'agresseur doit être confronté à son abus de pouvoir, doit reconnaitre la rentabilité de sa violence en réduisant ses rationnels de manipulation et doit développer un mode de comportement affirmatif, basé sur le respect de l'autre.

En tout temps, la protection de la victime doit être mise en première ligne de l'intervention. Mentionnons qu'il peut y avoir plus d'une victime lorsque la violence conjugale existe, les enfants en sont souvent témoins et victimes ${ }^{77}$ et la situation inverse est également vraie ${ }^{78}$.

Un ensemble de services à Montréal tente de répondre à cette réalité de violence. Voyons quels sont ces services.

\section{DISTRIBUTION RÉGIONALE DES SERVICES}

Depuis la mi-temps des années 70, la violence conjugale est sur la place publique. Les mouvements féministes ont brisé le silence. Au Québec, les maisons d'hébergement ont établi le premier sillon d'intervention auprès de cette clientèle. Plusieurs actions gouvernementales ont été mises de l'avant: 
colloque sur la violence en 1980 , politique familiale, politique d'aide aux femmes violentées, refonte du Code civil, politique judiciaire. Des formations dans le réseau et dans les universités ont été données. En plus des maisons d'hébergement à Montréal, des établissements reconnaissent et se préparent à offrir des services à cette clientèle: les CLSC, le $\operatorname{CSSMM}^{74}$.

La police de la CUM a maintenant des statistiques et un protocole de collaboration avec les CLSC est en cours. Une table de concertation en violence conjugale existe à Montréal. Bref, il y a eu depuis une douzaine d'années un ensemble d'actions pour identifier et nommer la violence conjugale. Les femmes battues ont recours à une dizaine de maisons d'hébergement à Montréal. Il existe plusieurs groupes d'aide aux hommes violents selon différentes écoles d'intervention (PRO-GAM, Option, Entre-Hommes, SAC, etc.). Certains groupes sont associés à des CLSC pour des services supports, d'autres sont indépendants.

Dans sa politique d'aide aux femmes violentées ${ }^{80}$, le MSSS reconnaît officiellement l'expertise et la place des maisons d'hébergement auprès de cette clientèle. Il reconnaît aussi l'importance des services pour hommes agresseurs. En plus, il propose des responsabilités à chaque catégorie d'établissements de son réseau, plus précisément les Conseils de la santé et des services sociaux (CRSSS), les Centres locaux de services communautaires (CLSC), les Centres de services sociaux (CSS) et finalement les Centres hospitaliers de soins de courte durée (CHSCD). Voici comment ces responsabilités se traduisent dans les différents secteurs concernés ${ }^{\mathrm{k}}$ :

Dans le réseau communautaire, les quatorze maisons d' hébergement, en plus d'assurer le gîte, offrent des services de relation d'aide, d'information et de référence, d'accompagnement, de suivi pré- et post-hébergement ainsi que des activités de sensibilisation et de prévention.

Les deux services pour hommes agresseurs interviennent auprès de leur clientèle par le biais d'évaluations et de consultations individuelles et de renconires de groupe. Des services d'information et de référence sont offerts aux conjointes et on dispense des programmes de formation pour les intervenants des différents réseaux concernés. En plus, un service d'aide aux conjoints offre de la relation d'aide, de l'écoute téléphonique, de l'information et de la référence.

De plus en plus, certains centres de femmes démontrent un intérêt pour les femmes violentées. Ceux-ci vont utiliser leurs services pour dépister ou encore pour accueillir les femmes et les intégrer à leurs activités et rencontres.

Dans le réseau de la santé et des services sociaux, le CSSSRMM tente d'assurer la coordination des services et la concertation des instances con- 
cernées par le biais du secteur de la Condition de vie des femmes, en collaboration avec les représentants de la Justice et de la Sécurité publique.

Les trente-trois CLSC, assurent à la communauté des services de prévention et d'actions sanitaires et sociales. Parmi les services offerts aux femmes violentées, nous retrouvons l'écoute téléphonique, des évaluations médicales ou psychosociales selon les besoins, des rencontres individuelles avec suivi à court terme, si la fermme le désire, un service de référence à d'autres services ainsi que des collaborations avec les services pour hommes agresseurs. Ces services sont disponibles aux heures d'ouverture du CLSC et c'est surtout à l'accueil et aux services courants que la demande est reçue.

D'une façon générale, les services dispensés par les CLSC varient beaucoup d'un établissement à l'autre et reposent bien souvent sur la présence et la disponibilité des ressources et des programmes.

Trois Centres de services sociaux desservent la région du Montréal métropolitain: le Centre de services sociaux du Montréal métropolitain (CSSMM) avec ses cinq bureaux, le Centre de services sociaux Ville-Marie (CSSVM) et le Centre de services sociaux juifs à la famille (CSSJF).

En ce qui a trait aux services aux femmes violentées, on note qu'une partie de leur personnel est sensibilisée à la problématique, que certain-e-s de leurs intervenant-e-s sont formé-e-s à l'approche féministe ou à l'intervention auprès des hommes agresseurs. Ceci se retrouve principalement au CSSMM De plus, celui-ci a envisagé des actions de recherche sur la violence faite aux personnes âgées et amorcé une réflexion sur une intervention spécifique auprès de cette clientèle.

Les CSS peuvent intervenir à différents niveaux auprès de cette clientèle:

- par des programmes ou interventions directes tels la consultation et le suivi psychosocial sur une base individuelle, ou encore par des rencontres de groupe;

- par le biais du Service de protection de la jeunesse (DPJ) où il est possible de faire du dépistage de situations de violence conjugale ainsi que de l'accueil, de la référence et un suivi à moyen et long terme pour la mère;

- par des interventions ponctuelles dans les services aux adultes et aux personnes âgées ou encore par celui aux migrants et aux immigrants;

- par le biais de l'urgence sociale, malgré que ce service ne reçoive que très peu de demandes. 
Tous les Centres de services sociaux offrent des services aux enfants abusés physiquement et sexuellement.

Vingt-six Centres hospitaliers de soins de courte durée desservent la région du Montréal métropolitain. Des intervenant-e-s de ces centres ont bénéficié de la formation «Accueil et dépistage» à l'automne 1987. Certains centres hospitaliers ont exprimé le besoin d'un protocole d'intervention pour l'accueil et le traitement des femmes violentées.

Cependant, nous possédons très peu d'information sur le degré de sensibilisation du personnel œuvrant auprès des femmes victimes de violence dans ces établissements. En général, on peut affirmer que les services médicaux sont les mêmes que ceux dispensés à toute personne se présentant à l'urgence, aux services externes ou à d'autres départements. Certains centres hospitaliers vont offrir des services de support et effectuer les références appropriées.

De plus, il est difficile de déterminer quel(s) groupe(s), parmi les professionnel-le-s œuvrant dans les centres hospitaliers, sont les plus actifs dans ce dossier. Il apparaît cependant que les infirmières et les travailleurs sociaux seraient les deux groupes les plus engagés. Nous possédons très peu d'information sur l'implication du secteur médical.

La problématique de la violence faite aux femmes et aux enfants n'est pas encore intégrée dans les services du secteur hospitalier. Par contre, les pratiques se développent et dans certains secteurs on retrouve de la recherche évaluative en regard de ces pratiques.

On peut conclure que la violence faite aux femmes et aux enfants est reconnue comme une réalité criante mais qu'elle ne fait pas encore l'objet d'interventions concertées dans l'ensemble des établissements de la région montréalaise.

\section{BILAN ET IMPACT DES ACTIONS PASSÉES - PRÉSENTES}

Des services sont donc disponibles sans nécessairement que ces ressources soient concertées et que les interventions soient bien identifiées et évaluées quant à leur pertinence. La violence est considérée comme un acte criminel sans que les victimes bénéficient d'un accompagnement psychosocio-judiciaire adéquat. Les femmes battues ne connaissent pas à Montréal des services d'aide complémentaires entre les organismes auxquels elles s'adressent. La politique d'aide aux femmes violentées n'opérationnalisait pas clairement les rôles entre les ressources de la communauté et celles du 
réseau parapublic. Cette confusion au niveau des rôles contribue à ralentir les tentatives de concertation.

Du côté de la recherche, plusieurs universités se sont penchées sur le phénomène de la violence. L'état de la question est fait. L'heure est à l'évaluation de modèles d'intervention, à l'élaboration, donc, d'interventions spécifiques $^{62}$. Toutefois, les connaissances sur l'intervention concernent davantage les victimes, le volet agresseur reste à évaluer. Ce dernier volet est encore à inscrire dans des démarches de recherche pour mieux le cerner.

Dans une perspective de monitoring du problème de la violence, celle-ci devrait être lue et traitée dans les différentes zones de victimisation.

En plus de ces impacts auprès des femmes violentées, il faut soulever le fait que la violence est lue de façon morcelée. En effet, les services s'impliquent en fonction de clientèles cibles: enfants battus, femmes battues, femmes violentées, personnes âgées. De façon systématique, il n'y a pas de dépistage et la lecture horizontale de la violence dans les différentes zones de victimisation ne se fait pas; malgré le fait qu'il est bien connu que les enfants battus vivent aussi dans un milieu de violence conjugale et que les personnes âgées sont aussi violentées par leurs enfants et inversement. La littérature permet de reconnaître qu'une victime, peu importe son statut dans la famille, vit des zones de dépendance qui la rendent vulnérable (tableau 3).

Il y aurait donc matière à développer des modèles d'intervention en violence quj tiendraient compte de certaines balises, ne serait-ce que de reconnaître les points communs chez les agresseurs et le vécu similaire des victimes. Ainsi les séquelles de la victimisation (perte d'estime de soi, diminution des habiletés affirmatives) auraient avantage à être traitées chez toutes les victimes indépendamment de leur âge. D’autre part, reconnaître qu'une victime risque de tolérer la violence si elle connaît plusieurs zones de dépendance permettrait aux intervenant-e-s d'offrir un accompagnement concret aux victimes. Finalement, reconnaître qu'une victime agit pour survivre permettrait aux praticien-nes de ne pas sous-estimer les capacités personnelles des victimes.

D'autre part, les agresseurs développent les mêmes rationnels pour faire usage de leur pouvoir et justifier leur violence. Si on se réfère au tableau 4 , on peut constater que les agresseurs ont des justifications de la violence qui se ressemblent peu importe le lien affectif qu'ils vivent avec leur victime.

Les données du tableau démontrent que les agresseurs tentent tous de justifier leur abus de pouvoir. Ils légitimisent leur violence en s'octroyant un rôle d'autorité sur la victime et en soutenant que ce privilège leur donne 
VIOLENCE FAITE AUX FEMMES

Zones de dépendancelzones de vulnérabilité se vivent différemment chez les victimes mais les exposent aux mêmes risques et augmentent leur tolérance à la violence.

\begin{tabular}{|c|c|c|}
\hline ENFANTS BATTUS & FEMMES BATTUES & PERSONNES ÂGÉES \\
\hline $\begin{array}{l}\text { - Le jeune enfant dépend émotivement et psy- } \\
\text { chologiquement de ses parents de par son statut } \\
\text { dans la famille } \\
\text { - Vit dans une famille isolée } \\
\text { - L'enfant qui est prématuré et qui a une santé } \\
\text { déficiente ou un retard mental } \\
\text { - L'enfant qui requiert des soins court plus de } \\
\text { risques d'être abusé lorsqu'il se retrouve avec } \\
\text { des parents potentiellement violents }{ }^{85}\end{array}$ & 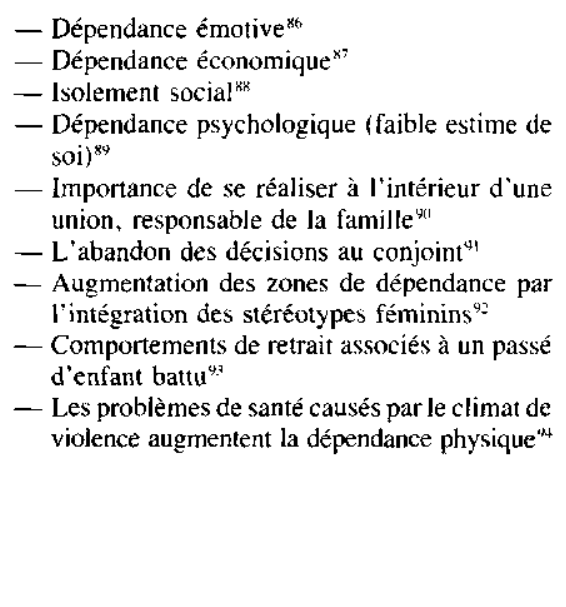 & $\begin{array}{l}\text { - } 13 \% \text { connaissent une dépendance économique } \\
\text { en vivant sous le seuil de la pauvretét } \\
\text { - État de santé précaire nécessitant des soins et } \\
\text { de l'attention (dépendance physique) } \\
\text { - Isolement social } \\
\text { - Dépendance émotive et psychologique }{ }^{97} \\
\text { - La vulnérabilité, la dépendance de la personne } \\
\text { âgée sont une cause d'abus physique }{ }^{98} \\
\text { - Les personnes dépendantes des autres pour } \\
\text { leurs besoins vitaux sont plus particulièrement } \\
\text { vulnérables }{ }^{99} \\
\text { - La dépendance vue comme une incapacité } \\
\text { expose la personne âgée à des sévices } \\
\text { violents } \\
\text { - } 51 \% \text { des personnes âgées admises dans un } \\
\text { hôpital pour chroniques ne pouvaient marcher } \\
\text { sans aide et avaient des problèmes sérieux de } \\
\text { santé, } 9 \% \text { de cette population subissaient des } \\
\text { violences }\end{array}$ \\
\hline
\end{tabular}

Plus il y a des zones de dépendance chez la victime, plus elle est vulnérable aux diverses formes de violence de l'agresseur et plus l'agresseur a du pouvoir. 


\begin{tabular}{|c|c|c|}
\hline HOMMES VIOLENTS & ENFANTS AGRESSEURS & PARENTS ABUSIFS \\
\hline $\begin{array}{l}\text { - La punition physique est vue comme un moyen } \\
\text { légitime de contrôler sa partenaire } \\
\text { - L'agression sert à prouver sa domination et à } \\
\text { établir son pouvoir } \\
\text { - L'isolement des membres de la famille aug- } \\
\text { mente son pouvoir } \\
\text { - L'agresseur blâme sa victime pour ses gestes } \\
\text { violents et la responsabilise de ses difficultés } \\
\text { - Les attentes sont irréalistes envers la } \\
\text { partenaire } \\
\text { - L'agresseur croit en la suprématie de } \\
\text { l'homme et a intégré les rôles traditionnels } \\
\text { la violence est alors un moyen pour contrôler } \\
\text { la vie de la famille } \\
\text { - La violence est perçue comme un moyen } \\
\text { acceptable de solutionner les conflits } \\
\text { - L'isolement des victimes demeure une stratégie } \\
\text { importante chez les agresseurs }\end{array}$ & $\begin{array}{l}\text { - Les adolescents recourent à la violence envers } \\
\text { leurs parents pour éviter leur contrôle }{ }^{112} \\
\text { - Refus de l'enfant de devenir une personne } \\
\text { aidante pour les parents (renversement des } \\
\text { rôles) } \\
\text { - L'adolescent veut contrôler sa famille } \\
\text { - L'adolescent qui veut établir sa suprématie } \\
\text { dans une relation prémaritale } \\
\text { - Les conflits de rôles entre les personnes âgées } \\
\text { et les enfants pour une lutte d'autorité } \\
\text { - La violence est perçue comme un moyen de } \\
\text { solutionner les conflits }\end{array}$ & 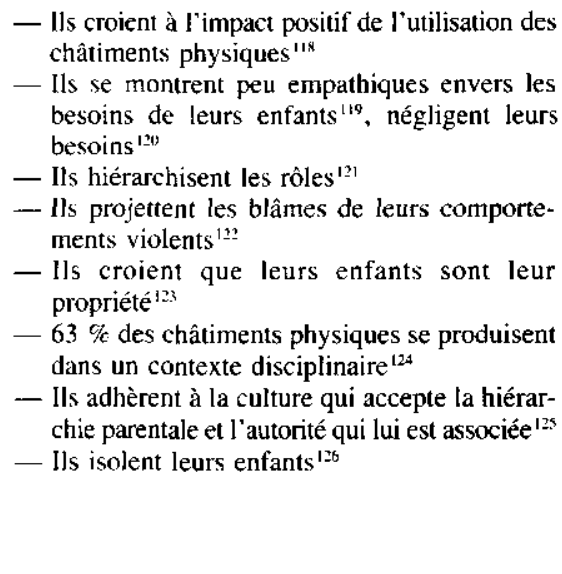 \\
\hline
\end{tabular}


le droit d'abuser des siens. Finalement, ils responsabilisent leur victime des comportements violents qu'ils commettent.

\section{CONCLUSION}

Les manifestations de la violence mobilisent sporadiquement le public lors d'événements tragiques tels les drames familiaux, les agressions collectives (École polytechnique) ou les gestes de «gangs» (agressions dans les autobus, mais cette mobilisation ne dure que le temps de l'événement. La violence fait l'objet de dénonciations dans ces périodes de crise mais cela ne suffit pas encore pour que notre communauté engage des actions concrètes et concertées pour diminuer cette violence.

Ainsi pour les années 90 , une perspective nouvelle se dégage dans l'intervention en matière de violence. Celle-ci s'oriente vers une analyse où la violence doit être lue dans toutes les sphères de victimisation. On parle maintenant de la violence conjugale, de celle faite aux femmes âgées. En plus de voir la situation des enfants battus, la réalité des enfants témoins de violence est aussi incluse dans les actions en matière de violence. Évidemment, les services aux hommes agresseurs s'inscrivent dans cette orientation. Des projets expérimentaux se vivent actuellement dans certains C.S.S. et dans quelques maisons d'hébergement où la violence est vue dans ses différentes manifestations. Sans tomber dans le piège de croire que la violence est une condition familiale, il s'avère pertinent de bien identifier chacune des victimes dans un milieu de violence, puisqu'il peut y en avoir plus d'une, et de reconnaître le pouvoir des agresseurs.

Une vision axée sur les besoins des victimes et qui tient compte de la nécessité d'intervenir auprès des agresseurs crée en partant une nécessité de concertation entre les ressources. Une volonté gouvernementale de partenariat peut s'inscrire dans une collaboration entre le ministère de la Justice et le ministère de la Santé et des Scrvices sociaux. De façon complémentaire, les établissements d'enseignement doivent s'inscrire rapidement dans ce mouvement.

La violence faite aux femmes et aux enfants ne pourra véritablement s'amoindrir tant que la valorisation des comportements violents demeurera présente dans les émissions de télévision; tant que la pornographie (violence aux femmes et aux enfants) sera présente dans les revues.

Il est nécessaire que des campagnes contre les comportements violents soient engagées. D'autre part, il est essentiel de former les médecins, les infirmières, les professeurs, les intervenants psychosociaux à dépister la violence. 
Une intervention précoce peut certainement réduire des escalades de violence et éviter des récidives.

La prévention passe également par un enseignement non sexiste dans les écoles afin d'éviter de reproduire des rapports de domination entre les hommes et les femmes.

En ce qui concerne l'intervention auprès des femmes violentées, il est maintenant temps d'évaluer les approches expérimentées depuis des années. De façon complémentaire, l'intervention disparate auprès des hommes violents aurait un urgent besoin d'être analysée et expérimentée par un accompagnement de recherche, de recherche-action, ou de recherche exploratoire.

Une démarche d'un tel ordre permettrait peut-être d'engager des échanges, entre les intervenants auprès des agresseurs et les intervenantes, tout en regroupant le nombre d'expérimentations auprès des agresseurs.

Finalement, la connaissance actuelle est assez vaste et précise pour que des interventions, expérimentées auprès des femmes violentées, soient analysées pour être essayées auprès des femmes âgées violentées. Dans toutes ces foulées, il ne faut pas oublier les enfants témoins et/ou victimes de violence. Ceux-ci font peu l'objet d'intervention. Ils sont protégés par la loi, mais peu d'intervenants travaillent directement avec les enfants sur les effets de leur vécu de violence.

Après dix ans d'expérimentation en violence envers les femmes en milieu familial à Montréal, il est temps de regarder comment établir des balises communes de travail qui pourraient bénéficier aux victimes et aux agresseurs.

Finalement, il est urgent de dépister et d'intervenir dans toutes les zones de victimisation dans le milieu familial.

\section{RÉFÉRENCES}

1. CARRIER, M., MICHAUD, M. (1982), La violence faite aux femmes en milieu conjugal: le produit d'une société sexiste. Étude québécoise, Secrétariat d'État, Ottawa.

MARTIN, G., LAVOIE, F. (octobre 1986), «Dilemmes et interventions dans le contexte de la violence conjugale», Interventions, no 75, 37-47.

MC LEOD, L. (1987), Pour de vraies amours... Prévenir la violence conjugale, Conseil consultatif canadien sur la situation de la femme, Ottawa.

LAROUCHE, G. (1985), Guide d'intervention auprès des femmes violentées, Corporation des travailleurs sociaux du Québec, Montréal.

2. MC LEOD dans la Commission d'enquête sur les services de santé et les services sociaux (1987), Dossier Femmes, Gouvernement du Québec, Québec. 
3. GUÈVREMONT, C., LAJEUNESSE, M., RONDEAU, G. (1986), "L'intervention auprès des hommes violents: le programme C.H.O.C.», Intervention. no 75 , 14-25.

4. ROSENBAUM, A., O'LEARY, D. (1981), «Marital violence: characteristics of abusive couples", Journal of consulting and clinical psychology, vol. 49, no 1, 63-71.

5. Solliciteur général du Canada (1985), «Le sondage canadien sur la victimisation en milieu urbain: les femmes victimes d'actes criminels», Bulletin, no 4, 4-9.

6. LAROUCHE, G., op.cit.

7. KEROUAC, M. N., TAGGARD, M. E., LESCOP, J. (1986), Portrait de la santé des femmes violentées et de leurs enfants, Conseil québécois de la recherche sociale, Montréal.

8. MC LEOD, L., CADIEUX, A. (1980), La femme battue au Canada, un cercle vicieux, Ottawa, Conseil Consultatif sur la situation de la femme.

DUPUIS, J., JOANNOU, S. (1982), Bibliographie annotée sur la violence conjugale. Santé et Bien-être social Canada.

9. DUPUIS, J. (1985), «L'urgence, le premier contact», Nursing Québec, vol. 5. no 5, 24-27.

10. FRANKEL-HOWARD, D. (1989), La violence familiale: examen des écrits théoriques et cliniques, Santé et Bien-être social Canada, Ottawa.

11. ROUNSAVILLE dans LAROUCHE, G. (1987), Agir contre la violence, La Pleine Lune.

12. DOBASH, R., DOBASH, R. E. (1980), Violence Against Wives, New York, Free Press.

13. BOURGON, M. (août 1988), Travail social et femmes de l'an 2000: les défis de l'avenir, texte non publié.

14. GUÈVREMONT, C., LAJEUNESSE, M., RONDEAU, G. (1986), «L'intervention auprès des hommes violents : le programme CHOC», Intervention, no 75, 14-25.

LAROUCHE, G. (1987), Agir contre la violence, La Pleine Lune.

MC LEOD, L., CADIEUX, A. (1980), La femme battue au Canada, un cercle vicieux, Ottawa, Conseil consultatif sur la situation de la femme.

15. RONDEAU, G. (1989), Les programmes québécois d' aide aux conjoints violents: rapport sur les 16 organismes existants au Québec. Planification, Évaluation. Santé et Services sociaux, Québec.

16. MARTIN, G., LAVOIE, F. (1986), «Dilemmes et interventions dans le contexte de la vioience conjugale", Interventions, no 75, 37-47.

PAQUET-DEEHY, A., RINFRET-RAYNOR. M., LAROUCHE, G. (1989), Apprendre à intervenir auprès des femmes violentées: une perspective féministe, Université de Montréal.

17. ROUNSA VILLE, B., LIFTON, N., BIEBER, M. (1979), «The natural history of a psychotherapy group for battered women", Psychiatry, vol. 42, 63-78.

18. HICKEY, T., DOUGLAS, R. (1981), «Neglect and abuse of older family members: Professionals' perspective». Gerontologist, vol. 21, no 2, 171-175.

19. SHELL, D. J. (1982), Protection des personnes âgées: étude sur les personnes âgées maltraitées. Manitoba Council on Aging.

20. GRANDMAISON, A. (1988), Protection des personnes âgées : étude exploratoire de la violence à l'égard de la clientèle des personnes âgées au CSSMM DSP. CSSMM. 
21. PEPPER dans SHELL, D. J., op.cit, no 19.

22. GOUVERNEMENT DU QUÉBEC (1989), Vieillir... en toute libertê : rapport du comité sur les abus exercés à l'endroit des personnes âgées, Gouvernement du Québec.

23. HÉTU, J. L. (1988), Psychologie du vieillissement, Méridien, Québec.

24. MAYER-RENAUD, M., TRÉPANIER, A. (1990), Réduire l'incidence et les conséquences des mauvais traitements et de la négligence envers les enfants, Document de consultation sur les objectifs de santé bien-être.

25. MARTIN, H. P. BEEZLEY, P., CONWAY, E., KEMPE, C. H. (1974), «The development of abused children", Advances in pediatrics, vol. 21, 25-73.

26. SINCLAIR, D. (1985), Understanding wife assault: a training manual for counsellors and advocates. Toronto. Ontario Ministry of Community and Social Services. Family violence program.

27. MARTIN et al., op. cit. no 24.

28. KÉROUAC, S., TAGGART, M. E., LESCOP, J., op. cit.

29. HOFELLER, K. (1982), Social Psychological Situational Factors in Wife Abuse, Californie, Palo Alto.

30. HILBERMAN dans LAROUCHE, G., op. cit. 14.

31. Commission d'enquête sur les services de santé et les services sociaux (1987). Programme de consultation d' experts, Dossier Femmes, Gouvernement du Québec.

32. Gouvernement du Québec (1985). Une politique d'aide aux femmes violentées. ministère des Affaires sociales. Québec.

33. LUPRI, E. (automne 1989), La violence masculine au fover. Tendances sociales cantdiennes, Statistique Canada, 19-20.

34. Gouvernement du Québec (1989), Statistiques 1988: violence conjugale, ministère de la Sécurité publique. Québec.

35. BARABÉ, L. (1989), Traitement des dossiers violence conjugale, Montréal.

36. BENNETT, C. (décembre 1989), Statistiques du SPCUM.

37. KOUSIK, J. (pour l'année 1988-89), Statistiques du SPCUM.

38. CAVAC (septembre 1989), Rapport d'étape du CAVAC.

39. BARIL, M., COUSINEAU, M. M., GRAVEL, S. (1983), «Quand les femmes sont victimes... quand les hommes appliquent la loi». Criminologie, 87-100.

40. ROSE, R. (10 décembre 1985), "Quelques statistiques sur la violence faite aux fèmmes et leurs coûts", La Presse.

41. PRUD'HOMME, Diane (novembre 1989), Derrière les chiffres 1988-89, Regroupement provincial des maisons d'hébergement.

42. Procès-verbal de la table de concertation sur la violence (8 Juin 1989), région de Montréal, rencontre.

43. CSSMM (1989), Rapport annuel.

44. MAKEPEACE, J. M. (1981), «Courtship violence among college students», Family relations, vol. 32, 101-109.

45. RONDEAU, G. (juin 1984), "La violence des hommes sur leur partenaire : causes et modèles d'intervention", Conférence-débat public, Montréal.

46. Assemblée des Évêques (1989), Violence en héritage, Comité des affaires sociales de l'Assemblée des Évêques, Montréal. 
47. FATTAH (1980), «Victimologie: tendances récentes», Criminologie, vol. 13, no 1 , 7-36.

48. ELBOW, M. (1977), «Theoretical considerations of violent mariages», Social casework, vol. $58,515-526$.

49. DELGATY, K. (1982), «Ces hommes qui maltraitent leur femme», Liaison, vol. 8, no $10,12-17$.

50. Idem.

51. RONDEAU, G., op. cit.

52. DANKWORTH, J. (1989), «Quatre conceptions de la violence conjugale : leur impact sur l'intervention auprès des hommes violents», Article soumis pour publication, Montréal.

53. ELBOW, M., op. cit.

54. WALKER, L. (1984), The Battered Women Syndrome, New York, Springer.

55. BUSCH, G., MOHR, V. (1980), «Evaluating child abuse intervention program». Social casework, vol. 61, no 2, 90-99.

56. MAKEPEACE, J. M., op. cit.

57. JOFFE, P., WOLF, D., WILSON, S. (1985), «Problèmes critiques mis en lumière par une étude sur l'adaptation des enfants témoins de violence dans la famille», Santé mentale au Canada, vol. 33, no 4, 14-18.

58. HARBIN, H. T., MADDEN, D. J., STEINMETZ, S. K. (1980). Behind Closed Doors; Violence in American Families, Garden City, New York. Anchor-Doubleday.

59. STRAUS, M. A.. GELLES, R. A., STEINMETZ, S. K. (1980), Behind closed doors: Violence in American Families, Garden City, New York. Anchor-Doubleday,

60. CORBEIL, C., PAQUeT-DeEHY, A., Lazure, C.. LegaulT, G. (1983), L'intervention féministe, I'alternative des femmes au sexisme en thérupie. Albert St-Martin, Montréal.

61. Idem

62. BALL, P. G., WYMAN, E. (1978), «Battered wives and powerlesness: what counselors do", Victimology, vol. 2, nos 3-4, 545-552.

63. JAKUBOWSKI, P. A. (1977), Psychotherapy for Women Treatment Toward Equality, Springfield, Ranling Carter, Charles Thomas Inc.

64. CORBEIL, C., PAQUET-DEEHY, A., LAZURE, C., LEGAULT, G., op. cit.

65. JAKUBOWSKI, P. A, op. cit.

66. HOFELLER, K. (1983), Battered women shattered lives, Paio Alto, California.

67. WALKER, L., op. cit.

68. HILBERMAN, E. (1980), «The wife beater's wife reconsidered», American journal of psychiatry, vol. 137, no 11

69. LAROUCHE, G., op. cit.

70. WALKER, L. E. (1979), The Battered Women, Harper \& Row, New York.

71. STEINMETZ, S. K. (1978), «Violence between family members», Marriage \& family review, vol. 1 , no 3, 1-16.

72. HILBERMAN, E., op. cit.

73. NICARTHY, G. (1983), Getting Free, Seal Press, Washington. 
74. STAR, B. (1978), «Comparing battered an non-battered wornen», Victimology vol. 2. no $3,32-34$.

75. HOFELLER, K., op.cit.

76. MC LEOD, L., op. cit.

76. MC LEOD, L., op. cit.

77. WALKER, L., op. cit.

78. SINCLAIR, D. (1985), Wife Assault : a Training Manual for Counsellors and Advocates, Ontario, Ministry of Social Services.

79. CSSMM (1989), La planification stratégique.

80. Gouvernement du Québec (1986). Une politique d'aide aux femmes violentées, ministère de la Santé et des Services sociaux, Québec, édition révisée.

81. GAGNÉ, L. LÉVESQUE, M. (janvier 1989), Répertoire des ressources pour l'accueil des personnes aux prises avec la violence, région de Montréal et Laval. CSSSRMM, éd. révisée.

82. RINFRET-RAYNOR, M., PAQUET-DEEHY, A., LAROUCHE G. (1989), Intervenir auprès des femmes violentées: évaluation de l'efficacité d'un modèle féministe: en cours actuellement.

83. BUSCH, G., MOHR, V., op. cit.

84. RUTH, S., KEMPE, H. (1987), L'enfant torturé, Édition Mardaga.

85. HELFER, R. E. (1982), «A Review of the literature on the prevention of child abuse and neglect», Child abuse and neglect, vol. 6, 251-261.

86. WALKER, L., op, cit.

87. STEINMETZ, S. K. (1977), The Cycle of Violence: Assertive, Agressive and Abusive Family Interaction, New York, Praeger.

88. HILBERMAN, E., MUNSON, L. (1977-78), «Sixty battered women», Victimology : an international journal, vol. 2, nos 3-4, 460-471.

89. CARLSON, B. (1977), «Battered women and their assailants», Social Work, vol. 22, no $61,455-460$.

90. NICARTHY, G. (1982), Getting Free, Seal Press, Washington.

91. STAR, B. op. cit.

92. BALL-WYMAN (1977-78), op. cit.

HOFELLER, K. (1982), Social Psychological Situational Factors in Wife abuse, Palo Alto, California.

93. LAROUCHE, G., op. cit.

94. HILBERMAN, E., op. cit.

KEROUAC, S., TAGGART, M. E., LESCOP, J., op. cit.

95. PILLEMER, K, (octobre 1987), «Les sévices aux personnes âgées sont un nouveau visage de la violence familiale», Actualité médicale, 11.

96. SHELL, D., op. cit.

97. PÉPIN, J., TAGGART, E., KÉROUAC, S., FORTIN, F. (1985), Étude systémique de la violence familiale, Montréal, Université de Montréal, 22-36.

STEINMETZ, S. K., op. cit. 
98. HICKEY, T., DOUGLASS, R. L. (1981), «Mistreatment of the elderly in the domestic setting: an exploraty study», American journal of public heath, no 71, 500-507.

PILLEMER, K., op. cit.

99. STEINMETZ, S. K., op. cit.

100. SHELL, D., op. cit.

101. LAU, E. (septembre-octobre 1979), "Provider", Aging.

102. DANKWORTH, J., op. cir.

103. ELBOW, M., op. cit.

104. PAHL dans KÉROUAC, S., TAGGART, M. E., LESCOP, J., op. cit.

105. LAROUCHE, G., op. cit.

DELGATY, K. (1982), «Ces hommes qui maltraitent leur femme», Liaison, vol. 8. no $10,4-9$.

106. RONDEAU, G. (juin 1984), «La violence des hommes sur leur partenaire féminin: causes et modèle d'intervention", Conférence-débat public, Montréal.

107. DOBASH R., DOBASH, R. E. (1980), Violence Against Wifes. New York, Free Press.

108. DELGATY, K., op, cit.

HOFELLER, K. (1982), Social Psychological Situational Factors in Wife Abuse, Palo Alto, California.

109. CARLSON, B., op. cit.

110. STRAUS, M. A., GELLES, R. A., STEINMETZ, S. K., op. cit.

111. HILBERMAN, E., op. cit.

112. LAROUCHE, G., op. cit.

113. SHELL, D., op. cit.

GRANDMAISON, A., op. cit.

114. HARBIN, H. T., MADDEN, D. J. (1979), «Battered parents: a new syndrome», American journal of psychiatry, no 136, 1288-1291.

115. WALKER, L., op. cit.

116. DAVIDSON dans PÉPIN, J., TAGGART, E., KÉROUAC, S., FORTIN, F., op. cit.

117. BANDURA. A. (1973), Agression : a Social Learning Analysis, New Jersey. Prentice Hall.

GELLES, R. J. (1980), «Violence in the family: a review of research in the seventies», Journal of Marriage and the family, vol. 2, no 42, 873-885.

118. BUSCH, G., MOHR, V., op. cit.

119. Idem.

120. MORRIS, M. B., GOULD, R. W. (I963), «Role reversal: a necessary conception dialing with the battered child's syndrome», American journal of orthopsychiatry, no $63,298-299$.

121. Parent and child therapy society (1984), «Learning and teaching in child abuse», Santé et Bien-être Canada.

122. Idem. 
123. GIL, D. G. (1976), «Primary prevention of child abuse a philosophical and political issue», Journal of pediatric psychology, vol. 1, 54-57.

124. BERGER dans PÉPIN, J., TAGGART, E., KÉROUAC, S., FORTIN, F., op. cit.

125. O'BRIEN, J. E. (1971), «Violence in divorce - prone families», Journal of marriage and the family, no. 33, 692-698.

126. YOUNG dans PÉPIN, J., TAGGART, E., KÉROUAC, S., FORTIN, F., op. cit. 